\title{
Changes in Body Color Appearance of the Black Tiger Prawn Penaeus monodon by the Varied Composition of Carotenoids Soluble as Carotenoprotein and Remaining Insoluble after Collagenase Treatment for the Muscular Epithelium
}

\author{
Shigeru Okada, Shah Amran Nur-E-Borhan, Shugo Watabe, \\ and Katsumi Yamaguchi
}

Laboratory of Marine Biochemistry, Faculty of Agriculture, The University of Tokyo, Yayoi, Bunkyo, Tokyo 113, Japan

(Received February 27, 1995)

\begin{abstract}
A blue carotenoprotein fraction was liberated from the muscular epithelium of the black tiger prawn Penaeus monodon by extraction with a collagenase solution, leaving another carotenoid fraction in the tissue. Carotenoids in both fractions were analyzed and compared between dark gray prawns and blue ones. The main carotenoid in the carotenoprotein was free astaxanthin, of which content showed no significant difference between the two groups. The muscular epithelium after the removal of blue carotenoprotein turned red owing to the remaining carotenoids which were composed largely of astaxanthin esters. The total content of the remaining red carotenoids was about six-fold higher in the dark gray than blue group. Thus the different body color of cultured black tiger prawns is suggested to be due to the varied composition of the blue carotenoprotein and red carotenoid fractions in the muscular epithelium.
\end{abstract}

Key words: black tiger prawn, body color, muscular epithelium, collagenase, carotenoprotein, astaxanthin

The black tiger prawn Penaeus monodon is one of the most important crustaceans in aquaculture among related species. The color of wild species is black or dark gray as indicated by its common name though blue prawns are often produced while cultured. This situation aroused us to carry out the first experiments for analyzing carotenoid content and composition in the exoskeleton of cultured specimens of $P$. monodon with different colors.") A dominant carotenoid was astaxanthin irrespective of the body color, though the total carotenoid content was markedly lower in blue prawns than in black or dark gray ones. Second, we have isolated and characterized blue carotenoproteins from the exoskeleton and the muscular epithelium of $P$. monodon, ${ }^{2)}$ These experiments were performed because crustacean carotenoids exist partly as a prosthetic group in carotenoproteins which generally exhibit a large bathochromic shift in the visible region of light absorption spectrum, giving rise to the expression of different colors; blue, ${ }^{3)}$ purple, ${ }^{4)}$ and yellow. ${ }^{5}$

In addition, penaeid prawns have very thin exoskeletons so that the color of the muscular epithelium can be seen without any anatomical treatment. Thus, the muscular epithelium highly contributes to the external appearance in terms of body color. This fact suggests that it is necessary to determine the types of carotenoids accumulated in both the muscular epithelium and the exoskeleton for elucidating the mechanism involved in body color development of penaid prawns. Although it was difficult to extract carotenoproteins quantitatively from the exoskeleton of $P$. monodon, we noticed that there was essentially no difference in the color tone between abdominal parts with and without the exoskeleton. Therefore, it seems reasonable to use those without the exoskeleton as materials for analyzing the types and quantities of carotenoid in relation to different colors developed in $P$. monodon.

In this study, we found two types of caroteoid accumulated in the muscular epithelium: one was water-soluble as carotenoproteins after collagenase treatment and the other remained in the tissue. The varied composition of these two fractions reflected different body color of $P$. monodon.

\section{Materials and Methods}

\section{Materials}

Frozen abdominal parts of the black tiger prawn $P$. monodon having two different colors, dark gray and pale blue, were purchased at the Tokyo Central Wholesale Market, and stored at $-80^{\circ} \mathrm{C}$ until used. Five prawns of both color types were used for analysis. The exoskeleton was removed from the abdominal muscle while they were partially frozen and the muscle part was subjected to the following experiments.

Extraction of Carotenoprotein from the Muscular Epithelium by Treatment with Collagenase

Collagenase (EC 3.4.24.3) from Clostridium histolyticum (Wako Pure Chemical, 208 units $/ \mathrm{mg}$ ) was dissolved at a concentration of $0.05 \%(\mathrm{w} / \mathrm{v})$ in $20 \mathrm{~mm}$ potassium phosphate buffer $(\mathrm{pH} \quad 6.8)$ containing $0.1 \mathrm{~mm}$ phenylmethylsulfonyl fluoride (PMSF). Prawns free from the exoskeleton were individually put into large mouth bot- 
tles with screw caps and to each specimen was added about $25 \mathrm{ml}$ of the collagenase solution to soak the abdominal part sufficiently. The bottles were incubated at $28^{\circ} \mathrm{C}$ for 3 $\mathrm{h}$ under gentle shaking, then a colored solution containing carotenoprotein was passed through No. 2 filter paper (Adavantec Toyo). The residual specimen was washed with a small volume of the buffer and the same procedure was repeated. The two successive fractions of the colored solution were combined and used for the following experiments together with the washed part.

\section{Determination of Carotenoid in the Carotenoprotein}

Carotenoids were liberated by adding acetone into the solution of carotenoproteins to a ratio of $20: 3$ by volume and partitioned between water and diethyl ether. ${ }^{6}$ The ethereal layer was evaporated under reduced pressure, then the carotenoids were dissolved in benzene and diluted to the volume. The total carotenoid content was determined at an absorption maximum around $480 \mathrm{~nm}$ by the method of McBeth with a Hitachi 330 spectrophotometer. ${ }^{7}$ The carotenoids containing astaxanthin derivatives were then subjected to silica gel column chromatography using a mobile phase of benzene/ethyl acetate $(9: 1, \mathrm{~V} / \mathrm{v})$ and separated into three fractions, including astaxanthin diester, astaxanthin monoester, and free astaxanthin. A relative composition of three fractions was determined spectrophotometrically.

\section{Determination of Carotenoids Remaining in the Muscular Epithelium}

Carotenoids remaining in the muscular epithelium after the removal of blue carotenoprotein by collagenase treatment were extracted with sufficient volumes of acetone. Extraction was repeated until the solution became colorless. The extracts were combined, evaporated under reduced pressure, and dissolved in benzene. Then the carotenoid content and relative composition of three forms of astaxanthin were determined as mentioned above.

\section{Statistics}

Statistical analyses were carried out by Student's $t$ test at a significant level of 1 or $5 \%$.

\section{Results}

Figure 1 shows the change of the color in the same prawns during extraction of carotenoprotein from the muscular epithelium by collagenase treatment. Figure 1a shows two groups of $P$. monodon with the exoskeleton; groups A and B for dark gray and blue prawns, respectively. The average weight for each of the two groups was almost the same (Table 1). Figure 1b shows the prawns without the exoskeleton. The color tone was so similar before and after removing the exoskeleton in both groups that the body color appearance seemed to be more related to the muscular epithelium than the exoskeleton. As reported in the preceding paper, ${ }^{2)}$ a carotenoprotein-containing solution was hardly obtained with different buffers often used for the extraction of carotenoproteins from the exoskeleton. Therefore, we assumed that carotenoproteins of $P$. monodon remained bound to the extracellular matrix of the muscular epithelium. Figure 1c shows the prawns after incubation with a solution containing collagenase. Blue carotenoproteins were extracted almost completely, and the specimens turned red probably due to the presence of other types of carotenoid remaining in the muscular epithelium. The red color of the specimen was clearly deeper in group A than in group B. In Fig. 2 is shown the blue carotenoprotein solution extracted with 20 mM phosphate buffer ( $\mathrm{pH}$ 6.8) containing collagenase. The color of the carotenoproteins extracted was not apparently different between both groups and from that of the exoskeleton extracted with an EDTA solution in our previous paper. ${ }^{2)}$

Table 1 shows the distribution of carotenoids in the muscular epithelium of the prawns for groups A and B. The content of carotenoid existing as carotenoprotein was not significantly different between both groups. On the other hand, the content of the carotenoids remaining in the muscular epithelium of group A was about six-fold higher than that of group B. These results were consistent with the difference in the appearance of the prawn color between both groups after collagenase treatment. Carotenoids remaining in the muscular epithelium were more abundant than those extracted as carotenoproteins for group A, whereas they were almost the same for the two corresponding fractions from group B. Table 2 shows relative composition of three forms of astaxanthin in the muscular epithelium. Free astaxanthin was a dominant form in the carotenoproteins for both groups. On the contrary, the carotenoids remaining in the muscular epithelium from both groups were composed primarily of astaxanthin esters; the main component in dark gray prawns was astaxanthin monoester, whereas it was astaxanthin diester in blue

Table 1. Distribution of carotenoids in the muscular epithelium

\begin{tabular}{|c|c|c|c|c|}
\hline \multirow{2}{*}{ Group } & \multirow{2}{*}{ Color } & \multirow{2}{*}{$\begin{array}{l}\text { Tail weight } \\
(\mathrm{g})^{* 1}\end{array}$} & \multicolumn{2}{|c|}{$\begin{array}{l}\text { Carotenoid content } \\
(\mu \mathrm{g} / \text { specimen })^{* 1,2}\end{array}$} \\
\hline & & & $\begin{array}{c}\text { Blue } \\
\text { carotenoprotein } \\
\text { fraction }\end{array}$ & $\begin{array}{l}\text { Red } \\
\text { carotenoid } \\
\text { fraction }^{* 3}\end{array}$ \\
\hline A & Dark gray & $23.8 \pm 0.6^{* 4}$ & $68.6 \pm 27.3^{* 4}$ & $252.4 \pm 75.6^{* 5}$ \\
\hline $\mathrm{B}$ & Blue & $23.4 \pm 1.8$ & $45.9 \pm 8.9$ & $40.6 \pm 8.3$ \\
\hline
\end{tabular}

*1 Mean \pm standard deviation $(n=5)$

$*_{2}$ In terms of free astaxanthin.

${ }^{*}$ Carotenoids remaining in the muscular epithelium after collagenase treatment.

$*_{4}$ The difference between groups A and B is not significant $(p>0.05)$.

$*_{5}$ The difference between groups $\mathrm{A}$ and $\mathrm{B}$ is significant $(p<0.01)$.

Table 2. Relative composition of three forms of astaxanthin in the muscular epithelium

\begin{tabular}{|c|c|c|c|c|}
\hline \multirow{2}{*}{ Color } & \multirow{2}{*}{ Accumulation type } & \multicolumn{3}{|c|}{ Astaxanthin form $(\%)^{* i}$} \\
\hline & & Diester & Monoester & Free \\
\hline \multirow{2}{*}{$\begin{array}{l}\text { Dark } \\
\text { gray }\end{array}$} & \multirow{2}{*}{$\begin{array}{l}\text { Blue carotenoprotein fraction } \\
\text { Red carotenoid fraction }\end{array}$} & $16 \pm 3^{* 2}$ & $21 \pm 6^{* 3}$ & $63 \pm 7^{* 4}$ \\
\hline & & $37 \pm 2^{* 5}$ & $54 \pm 5^{*}$ & $9 \pm 3^{* 7}$ \\
\hline \multirow{2}{*}{ Blue } & \multirow{2}{*}{$\begin{array}{l}\text { Blue carotenoprotein fraction } \\
\text { Red carotenoid fraction }\end{array}$} & $10 \pm 1^{*_{2}}$ & $3 \pm 2^{* 3}$ & $87 \pm 2^{* 4}$ \\
\hline & & $54 \pm 8 * 5$ & $14 \pm 2^{*_{6}}$ & $32 \pm 7^{* 7}$ \\
\hline
\end{tabular}

$*_{1}$ Mean \pm standard deviation $(n=5)$.

$*_{2,3,4,5,6,7}$ The differences between the values with same number are siginificant $(p<0.01)$ 


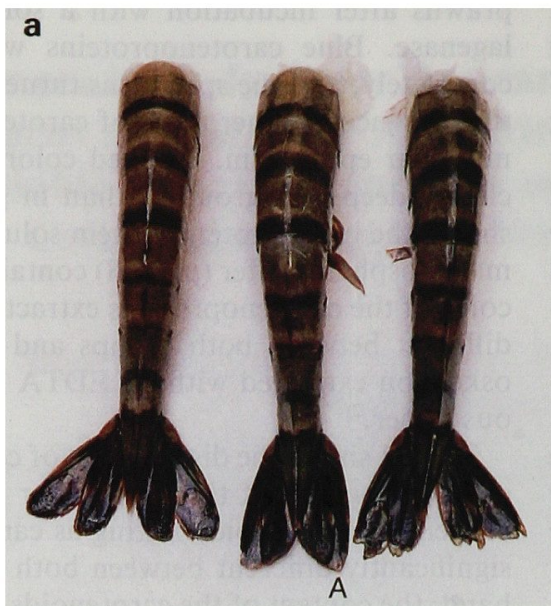

b

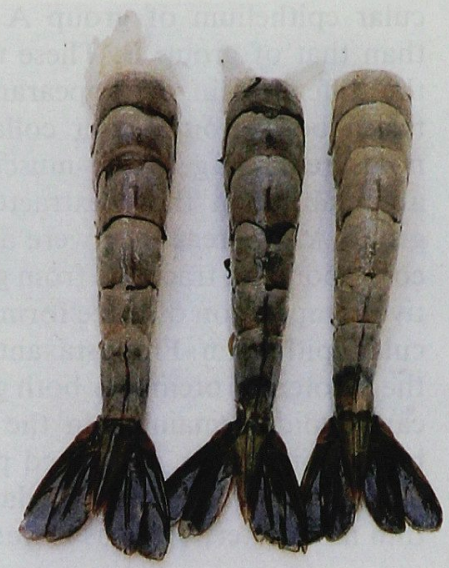

A

C

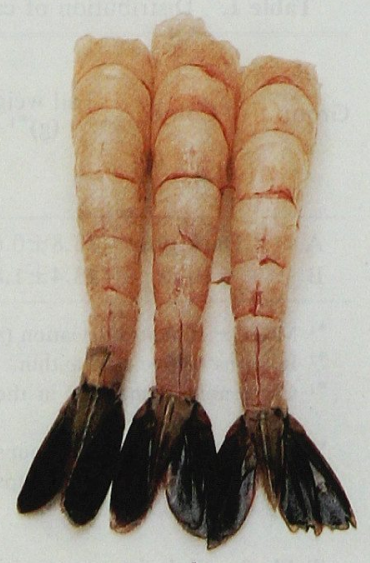

A

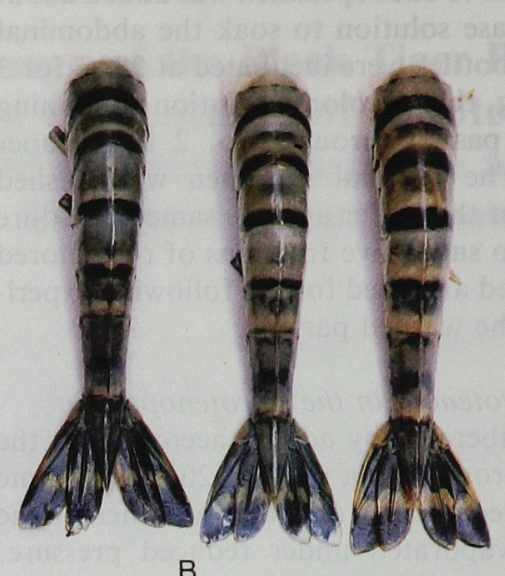

B
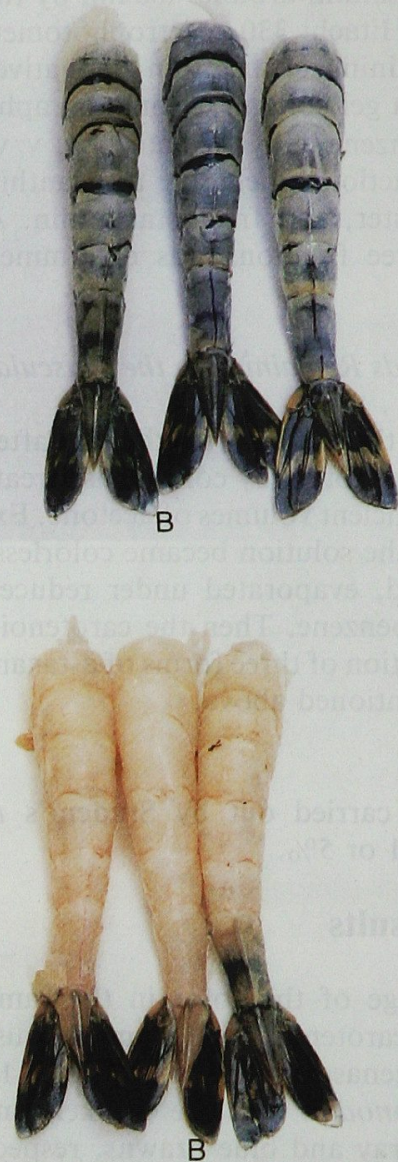

Fig. 1. Penaeus monodon of two color types.

a: Prawns with the exoskeleton. b: Prawns without the exoskeleton. c: Prawns without the exoskeleton after removing blue carotenoproteins by collagenase treatment. Groups A and B represent dark gray and blue prawns, respectively.

prawns.

\section{Discussion}

In our previous studies, we found that purified carotenoproteins from the exoskeleton and muscular epithelium of $P$. monodon contained only free astaxanthin as a prosthetic group. ${ }^{2)}$ In this study, however, extracted carotenoproteins by collagenase treatment contained not only free astaxanthin but also small amounts of astaxanthin esters. These astaxanthin esters were supposedly extracted from the red carotenoids deeply seated in the mus- 


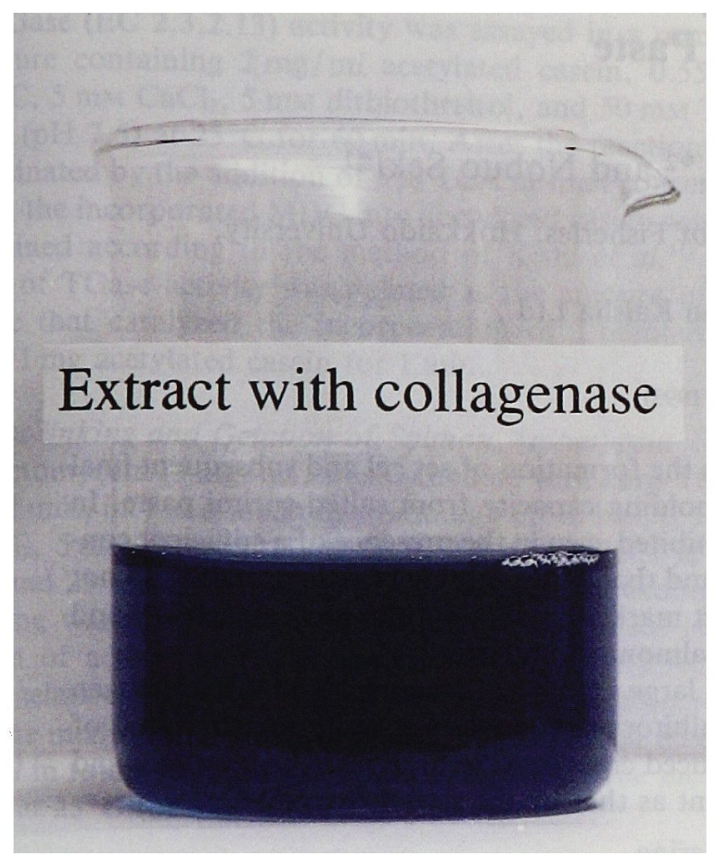

Fig. 2. The blue carotenoproteins extracted with a solution containing collagenase.

cular epithelium during collagenase treatment, which might loosen the tissue structure surrounding accumulated carotenoids. This assumption was supported by the fact that the carotenoid content as well as the percentage of astaxanthin esters in the blue carotenoprotein fraction was higher in dark gray than blue prawns (Tables 1 and 2). When astaxanthin content in the blue carotenoprotein fraction is revised according to supposition that all esterified astaxanthin detected in carotenoprotein solution was derived from carotenoids deeply situated in the muscular epithelium, free astaxanthin contents, 43.2 and 39.9 $\mu \mathrm{g} /$ specimen for groups $\mathbf{A}$ and $\mathbf{B}$, respectively, in the form of carotenoprotein in both groups become converged.

From these results, it seems that there is a limitation in the muscular epithelium for the maximal accumulation of free astaxanthin as carotenoproteins extractable by collagenase treatment. A similar tendency can be seen in the carotenoid composition of the exoskeleton of $P$. monodon with different colors, where all free astaxanthin is likely to exist as easily soluble carotenoprotein. ${ }^{1)}$ In this connection, Howell and Matthews claimed that farmed specimens of $P$. monodon with blue color contained only a small amount of astaxanthin esters. ${ }^{8)}$ Yamada et al. ${ }^{9)}$ reported that $P$. japonicus accumulated astaxanthin as esters when fed a sufficient amount of carotenoids in diets, and that the level of free astaxanthin was not influenced by the dietary carotenoid level. Penaeid prawns may accumulate dietary carotenoids as carotenoprotein complexed with free astaxanthin to a certain level, then store excess carotenoids after converting them to astaxanthin esters.

Little difference in the free astaxanthin content in blue carotenoprotein between blue and dark gray prawns suggests that the dark gray color is not owing to a higher accumulation of the blue carotenoprotein. Dark gray prawns contained the large quantities of astaxanthin esters deeply seated in the muscular epithelium, so that the blue color developed by carotenoproteins is rather declined. On the other hand, the red color due to the abundance of astaxanthin esters is responsible for the dark gray color when mixed with the blue color caused by the presence of blue carotenoproteins. The color development must be largely affected by the composition of carotenoids containing free astaxanthin and astaxanthin esters which show anatomical localizations (see Figs. 1a-c). Thus, black or dark gray color of the muscular epithelium of prawns is expressed by the overlapping of blue carotenoproteins and deeply seated red carotenoids. In addition, the exoskeleton that has almost the same color tone and carotenoid distribution as those of the muscular epithelium may have a role to make the body color clearer by shielding the color of the muscular epithelium.

Acknowledgments This study was partly funded by a Grant-in-Aid from the Ministry of Education, Science, and Culture of Japan.

\section{References}

1) S. Okada, S. A. Nur-E-Borhan, and K. Yamaguchi: Carotenoid composition in the exoskeleton of commercial black tiger prawns. Fisheries Sci., 60, 213-215 (1994).

2) S. A. Nur-E-Borhan, S. Okada, S. Watabe, and K. Yamaguchi: Carotenoproteins from the exoskeleton and the muscular epithelium of the black tiger prawn Penaeus monodon. Fisheries Sci., 61, 337343 (1995).

3) D. F. Cheesman, P. F. Zagalsky, and H. J. Ceccaldi: Purification and properties of crustacyanin. Proc. Roy. Soc., London, B164, 130-151 (1963).

4) R. Gomez, I. Manzano, A. M. Garate, P. G. Barbon, J. M. Macarulla, and J. C. G. Milicua: A purple carotenoprotein from the carapace of Galathea strigosa. Comp. Biochem. Physiol., 90B, 53-57 (1988).

5) M. Buchwald and W. P. Jencks: Properties of the crustacyanins and the yellow lobster shell pigfient. Biochemistry, 7, 844-859 (1968).

6) R. Powl and G. Britton: A carotenoprotein containing violaxanthin isolated from Scenedesmus obliquus $\mathrm{D}_{3}$. Biochim. Biophys. Acta, 453, 270-276 (1976).

7) J. W. McBeth: Carotenoid from nudibranchs. Comp. Biochem. Physiol., 41B, 55-68 (1972).

8) B. K. Howell and A. D. Matthews: The carotenoids of wild and blue disease affected farmed tiger shrimp (Penaeus monodon, Fabricus). Comp. Biochem. Physiol., 98B, 375-379 (1991).

9) S. Yamada, Y. Tanaka, M. Sameshima, and Y. Ito: Pigmentation of prawn (Penaeus japonicus) with carotenoids. I. Effects of dietary astaxanthin, $\beta$-carotene and canthaxanthin on pigmentation. Aquaculture, 87, 323-330 (1990). 